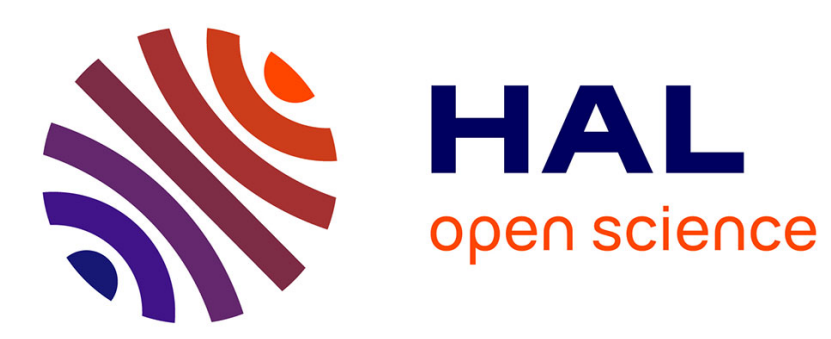

\title{
Influence of Beamforming Methods on Velocity Estimation: in vitro Experiments
}

\author{
M. Polichetti, Vincent Perrot, H. Liebgott, B. Nicolas, F. Varray
}

\section{To cite this version:}

M. Polichetti, Vincent Perrot, H. Liebgott, B. Nicolas, F. Varray. Influence of Beamforming Methods on Velocity Estimation: in vitro Experiments. 2018 IEEE International Ultrasonics Symposium (IUS), Oct 2018, Kobe, Japan. 10.1109/ULTSYM.2018.8580186 hal-01894167

\section{HAL Id: hal-01894167 https://hal.science/hal-01894167}

Submitted on 11 Mar 2019

HAL is a multi-disciplinary open access archive for the deposit and dissemination of scientific research documents, whether they are published or not. The documents may come from teaching and research institutions in France or abroad, or from public or private research centers.
L'archive ouverte pluridisciplinaire HAL, est destinée au dépôt et à la diffusion de documents scientifiques de niveau recherche, publiés ou non, émanant des établissements d'enseignement et de recherche français ou étrangers, des laboratoires publics ou privés. 


\title{
Influence of Beamforming Methods on Velocity Estimation: in vitro Experiments
}

\author{
Maxime Polichetti*†, Vincent Perrot*, Hervé Liebgott*, Barbara Nicolas* \\ and François Varray* \\ ${ }^{*}$ CREATIS, Univ. Lyon, INSA-Lyon, Université Claude Bernard Lyon 1, UJM-Saint-Etienne, \\ CNRS, Inserm, CREATIS UMR 5220, U1206, Lyon, France \\ Email: maxime.polichetti@creatis.univ-lyon1.fr, vincent.perrot@creatis.univ-lyon1.fr \\ †Université Lyon, Inserm U1032, LabTau, Université Claude Bernard Lyon 1, Lyon, France
}

\begin{abstract}
Many beamforming methods have been developed for ultrasound B-mode imaging, as an alternative to conventional Delay-And-Sum (DAS) algorithm. Fourier-based methods decrease the computational load, while adaptive ones enhance Bmode image quality. These beamformers were mainly evaluated using image quality metrics on static media, such as resolution and contrast, but never on motion estimation.

Herein, the influence of six beamformers on velocity estimation is quantitatively evaluated experimentally with a rotating disk. The conventional DAS was compared with several beamformers introduced in the literature: two Fourier-based techniques (Lu's and $f-k$ ), and three adaptive ones (Minimum Variance (MV), Phase Coherence Factor (PCF) and DAS with p-th root compression of signals (p-DAS)). The experimental results demonstrate that advanced beamformers are compatible with velocity estimation. Fourier-based methods slightly outperformed DAS, and adaptive beamformers can offer comparable velocity maps as DAS with similar statistics regarding bias and standard deviation. However, their performances decreased when increasing the adaptive effect.
\end{abstract}

\section{INTRODUCTION}

In medical ultrasound, ultrafast plane wave imaging has been a real breakthrough [1]. The conventional frame rate has been increased form some tens to thousands image per second. The plane wave imaging has enabled the emergence of many applications for the observation of transient biological phenomena [2].

Also, new image reconstruction algorithms have been developed, with the emergence of plane wave imaging. Methods have been proposed to reduce the computation load of the conventional Delay-And-Sum (DAS) beamforming operating in the time-domain. Thanks to Fourier-based approaches, Lu's method [3] and Stolt's $f-k$ migration [4] offer similar or even better B-mode image quality concerning resolution and contrast.

However, image quality with plane wave is degraded with respect to conventional multi-focused one. Then many adaptive beamforming technics have been proposed to preserve both resolution and contrast of B-mode images with a high frame rate [5]. The Capon's Minimum Variance beamforming (MV) has been adapted to medical ultrasound from acoustic imaging [6]. Other beamformers aim to increase the effect of coherent summation along the element dimension involved in the conventional DAS. In [7], the resolution and contrast are enhanced thanks to a non-linear amplitude compression of acquired signals (pDAS beamforming), whereas in [8], the coherent summation is reinforced thanks to a phase-based dispersion criterion (PCF: phase coherence factor).

However, from our knowledge, such methods (migration or adaptive technics) have only been tested according to Bmode image quality metrics. Since the velocity estimation is based on the RF-images after beamforming, the choice of the beamformer necessarily influences the quality of velocity estimation. This study proposes to experimentally quantify the influence of advanced beamformers on velocity estimation, considering a rotating agar gel disk. Two advanced migration methods (Lu's and $f-k$ ), and three adaptive beamformers (MV, pDAS, and PCF) are compared to conventional DAS.

The paper is organized as follows. The second section describes the six compared beamformers, the method for velocity estimation, and the experimental setup. The third section analyzes the results. The paper ends with a discussion and conclusions.

\section{Methods AND MATERIALS}

The Figure 1 provides the global pipeline of the study. A rotating disk is imaged using an unsteered single plane wave. Then, the raw data are beamformed into an RF image. Two consecutive RF images are used to obtain an instantaneous velocity estimation. The statistics of estimates are computed from 253 maps (which corresponds to around $250 \mathrm{~ms}$ of data acquisition). The velocity estimation method is unchanged, whereas six different beamformers are investigated.

\section{A. Beamforming methods}

This section describes the conventional DAS beamformer, three adaptive ones (MV, PCF, pDAS), and two advanced migration technics (Lu's, $f-k$ ).

A linear probe of $N$ elements is used for both transmission and reception. The reconstruction grid is $(x, z)$, where $x$ is the lateral axis parallel to the array, and $z$ is the depth axis.

1) Conventional Delay-And-Sum beamforming (DAS): Each pixel $r_{D A S}(x, z)$ of the RF image is obtained through a combination of the $N$ correctly delayed samples $s_{n}(x, z)$, extracted from the raw echo signals $p_{n}(t)$, where $n$ is the element index. Two assumptions are made: the speed of sound 


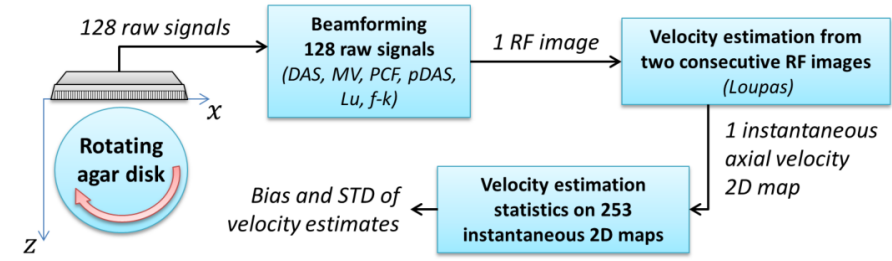

Fig. 1. Global block diagram of the study. A rotating disk is imaged using an unsteered single plane wave. Then, the raw data are beamformed into an $\mathrm{RF}$ image. Two consecutive RF images are used to obtain an instantaneous velocity estimation. The statistics of estimates are computed from 253 maps (which corresponds to around $250 \mathrm{~ms}$ of data acquisition). The velocity estimation method is unchanged, whereas six different beamformers are investigated.

in the phantom is homogeneous $c_{0}=1540 \mathrm{~m} \cdot \mathrm{s}^{-1}$, and a point scatterer generates a spherical wavefront. In this way, the echo back-scattered by a point located at $(x, z)$ corresponds to the samples $s_{n}(x, z)$ with:

$$
s_{n}(x, z)=p_{n}\left(\tau_{n}(x, z)\right),
$$

where $\tau_{n}(x, z)$ is the time of flight of the wave, which is the sum of the time in transmission for the plane wave to get to the scatterer, and the time in reception from the scatterer to element $n$ of the probe:

$$
\tau_{n}(x, z)=\frac{1}{c_{0}}\left(z+\sqrt{\left(x-x_{n}\right)^{2}+z^{2}}\right),
$$

where $x_{n}$ is the lateral position of the $n^{\text {th }}$ element. Finally, the samples $s_{n}(x, z)$ are merely summed along the element dimension, to obtain the RF image pixel value $r_{D A S}(x, z)$ :

$$
r_{D A S}(x, z)=\sum_{n=1}^{N} a_{n} \cdot s_{n}(x, z),
$$

where $a_{n}$ are the weighting coefficients of the apodization window (e.g., Tukey, Hann, and others). For DAS, a uniform apodization is used, with a constant F-number of 1.5.

The three adaptive beamformers of this study (MV, pDAS, PCF) follow the same process for delaying as the DAS, with a constant F-number of 1.5. The difference lies in the way $s_{n}(x, z)$ are combined to obtain the pixel value.

2) Minimum Variance beamforming (MV): For each pixel value, the Capon's Minimum Variance beamforming (MV) computes the set of adaptive weights that minimizes the power of equation (3), with a constraint of unity gain on the pixel of interest [6]. This method requires the inversion of the spatial covariance matrix $R\left(x_{0}, z_{0}\right)$ of the rephased samples, which is not necessary full-ranked. Then, the MV needs two operations to be more robust. First, the spatial smoothing rearranges the $N$ samples $s_{n}\left(x_{0}, z_{0}\right)$ into $N-L+1$ subarrays of width $L$, to provide an estimate $\hat{R}\left(x_{0}, z_{0}\right)$ of $R\left(x_{0}, z_{0}\right)$. Second, the diagonal loading consists of forcing $\hat{R}\left(x_{0}, z_{0}\right)$ as a full-ranked matrix before its inversion. A coefficient $\delta$ is added on its diagonal, which corresponds to a spatially uncorrelated white noise with a power $\delta$ [6]. Finally, the RF image returned by the MV beamforming is:

$$
r_{M V}\left(x_{0}, z_{0}\right)=\frac{1}{N-L+1} \sum_{l=1}^{N-L+1} \mathbf{w}^{H}\left(x_{0}, z_{0}\right) \tilde{\mathbf{s}}_{l}\left(x_{0}, z_{0}\right)
$$

with $\tilde{\mathbf{s}}_{l}\left(x_{0}, z_{0}\right)$ is the $(L, 1)$-vector corresponding to the $l^{\text {th }}$ smoothed subarray, w is the $(L, 1)$-vector corresponding to the set of adaptive weights, $(.)^{H}$ denotes the conjugate transpose, $L$ and $\delta$ are two user parameters. As $L$ increases, the adaptive effects of MV increase too (until maximum value $L=N / 2$ ). If $L=1$ the RF image obtained tends to the DAS one. In this study, $\delta$ was empirically set constant to $\delta=1000$.

3) Phase Coherence Factor beamforming (PCF): In [8], the phase coherence factor (PCF) was proposed to attenuate pixel values resulting from incoherent summation in equation (3). This factor is computed from the phase variance $\sigma_{\phi}\left(x_{0}, z_{0}\right)$ of each set of $N$ delayed samples $s_{n}\left(x_{0}, z_{0}\right)$, on the element dimension $n$, as:

$$
w_{P C F}\left(x_{0}, z_{0}\right)=\max \left[0 ; 1-\frac{\gamma}{\sigma_{0}} \sigma_{\phi}\left(x_{0}, z_{0}\right)\right],
$$

where $\gamma$ is a user parameter (default value is 1), and $\sigma_{0}=$ $\frac{\pi}{\sqrt{3}}$ is a normalization factor corresponding to the standard deviation of a uniform phase distribution. The $\gamma$ value can accentuate the adaptive effects or it can be set to 0 to recover conventional DAS.

4) Nonlinear beamforming based on p-th root compression (pDAS): In [7], the coherent summation in equation (3) is reinforced by averaging the signed $p$-th root of delayed signals:

$$
\tilde{r}_{p D A S}\left(x_{0}, z_{0}\right)=\sum_{n=1}^{N} \operatorname{sign}\left(s_{n}\left(x_{0}, z_{0}\right)\right)\left|s_{n}\left(x_{0}, z_{0}\right)\right|^{1 / p} .
$$

Then, as the $p$-th root compression changes the dimension of data (e.g., from volts to volts ${ }^{1 / p}$ ), the signed $p$-power is applied to the resulting sum:

$$
r_{p D A S}\left(x_{0}, z_{0}\right)=\operatorname{sign}\left(\tilde{r}_{p D A S}\left(x_{0}, z_{0}\right)\right)\left|\tilde{r}_{p D A S}\left(x_{0}, z_{0}\right)\right|^{p} .
$$

The reconstructed image must be band-pass filtered (along the $z$ dimension) around the transmit frequency to remove potential artificial harmonics induced by nonlinear operations [7]. The $p$ value is the user parameter to accentuate the adaptive effects or it can be set to 1 to recover DAS.

5) Lu's and Stolt's $\boldsymbol{f}$ - $\boldsymbol{k}$ migrations: The Lu's [3] and Stolt's $f$ - $k$ [4] beamformers are not adaptive beamformers, but advanced migration methods. They aim to reduce the computing time of DAS, by operating in the Fourier domain. They consist of a frequency remapping of the two dimensional fast Fourier transform of the $N$ raw signals. The Lu's method is based on a scattering model, whereas the $f-k$ one is instead based on a reflector model. They slightly differ on the way the frequency are remapped, as explained in details in [4]. 


\section{B. Velocity estimation}

The velocity estimation consists of a $2 \mathrm{D}$ map of axial velocity, from two successive beamformed RF images, acquired with a frame rate of $P R F=1 \mathrm{kHz}$. A stack of $254 \mathrm{RF}$ images has been beamformed, giving 253 instantaneous axial velocity $2 \mathrm{D}$ maps across the time $v_{z}(x, z)$. Each resulting $2 \mathrm{D}$ map $v_{z}(x, z)$ is estimated using the complex autocorrelation function as in [9], [10], after I/Q demodulation at the transmit frequency $f_{0}$ :

$$
v_{z}(x, z)=-\frac{c_{0} \cdot P R F}{4 f_{0}} \frac{\angle \hat{R}(1)(x, z)}{\pi}
$$

where $c_{0}$ is the speed of sound $(\mathrm{m} / \mathrm{s})$ and $\angle \hat{R}(1)(x, z)$ is the angle of the complex autocorrelation function at lag 1 . A spatial average with a 2D Hamming kernel (size: $x=1$ $\mathrm{mm}, z=2.5 \mathrm{~mm}$ ) is applied on the complex autocorrelation.

\section{Experimental setup}

An agar and silica-based rotating disk with a radius of $15 \mathrm{~mm}$ was imaged at different constant velocities 50, 100 and $150 \mathrm{deg} / \mathrm{s}$ (which corresponds respectively to a maximum velocity at the edge of the disk of $13 \mathrm{~mm} / \mathrm{s}, 26 \mathrm{~mm} / \mathrm{s}$, and $38 \mathrm{~mm} / \mathrm{s}$ ). A Verasonics ultrasound system (Verasonics Inc., Redmond, WA) and the L7-4 probe were used. The acquisition parameters are described in Table I.

TABLE I

ACQUISITION PARAMETERS

\begin{tabular}{|c|c|}
\hline Pitch & $298 \mu \mathrm{m}$ \\
\hline Number of elements & $128(\mathrm{Tx} / \mathrm{Rx})$ \\
\hline Sampling frequency & $20.8 \mathrm{MHz}$ \\
\hline Speed of sound & $1540 \mathrm{~m} / \mathrm{s}$ \\
\hline Pulse repetition frequency & $1 \mathrm{kHz}$ \\
\hline Transmission mode & Single plane wave (unsteered) \\
\hline Transmit frequency & $5.2 \mathrm{MHz}$ \\
\hline Transmit pulse & $3 \mathrm{cycles}$ \\
\hline Transmit apodization & Tukey $(25 \%)$ \\
\hline Receive apodization & Rectangular \\
\hline
\end{tabular}

\section{RESUlts}

In this section, the results obtained with DAS, Lu's, $f-k$, MV, pDAS, and PCF beamforming are presented: the Bmode images and the instantaneous $2 \mathrm{D}$ velocity maps are qualitatively discussed, and then a quantitative analysis of the velocity estimation statistics is presented.

\section{A. B-mode analysis}

The B-mode images of the rotating disk at the constant velocity of $100 \mathrm{deg} / \mathrm{s}$, obtained for the $100^{t h}$ acquisition, are shown in Figure 2. To show the effect of each adaptive beamformers, two values of user parameters are investigated. For MV, as the subaperture width $L$ decreases the grains in the speckle are more prominent, in Figure 2(d) $L=0.3 \mathrm{~N}$ and (e) $L=0.5 N$. For pDAS (respectively, PCF), the higher the value of $p$ (respectively, $\gamma$ ), the darker the outside of the disk and the higher the variance of the gray level inside the disk, as shown in Figures $2(\mathrm{f}) p=1.2$ and $(\mathrm{g}) p=2$ (respectively, (h) $\gamma=1$ and (i) $\gamma=1.15$ ). Considering advanced migration technics, (b) Lu's and $f-k$ (c) methods offer similar B-mode images as (a) DAS, except in the near field: for Lu's and $f-k$, the background outside the disk is a little bit more noisy, since they do not benefit from the constant F-number as DAS.

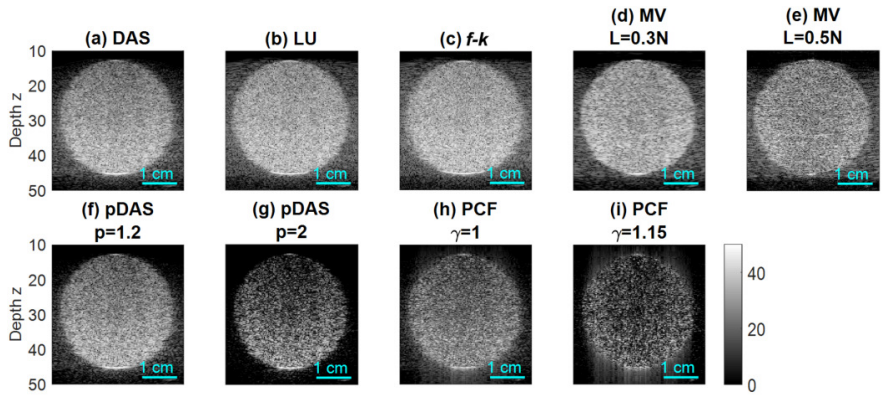

Fig. 2. B-mode images of the rotating disk at the constant velocity of 100 $\mathrm{deg} / \mathrm{s}$, obtained for the $100^{t h}$ acquisition with different beamformers. For each of the three adaptive beamformers, two values of adaptive parameter are presented.

\section{B. Influence of beamforming methods on velocity estimation}

1) Qualitative analysis: The instantaneous 2D axial velocity maps of the rotating disk at the constant velocity of 100 $\mathrm{deg} / \mathrm{s}$, obtained for the $100^{\text {th }}$ and $101^{\text {st }}$ acquisitions are shown in Figure 3. To show the effect of each adaptive beamformers, two values of user parameters are investigated as for Bmodes. For all adaptive beamformers, as their respective user parameter values decrease, the velocity maps are smoother and closer to the ground truth in (j) (see Figure 3, for MV (d) $L=0.3 N$ and (e) $L=0.5 N$, for pDAS (f) $p=1.2$ and (g) $p=2$, for PCF (h) $\gamma=1$ and (i) $\gamma=1.15$ ). Considering advanced migration technics, (b) Lu's and (c) $f$ - $k$ methods offer similar velocity estimates as (a) DAS.
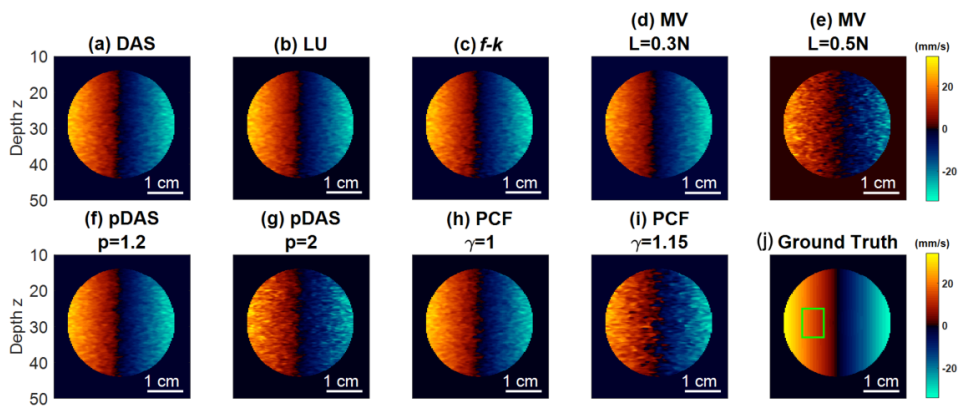

Fig. 3. Instantaneous $2 \mathrm{D}$ axial velocity maps of the rotating disk at the constant velocity of $100 \mathrm{deg} / \mathrm{s}$, obtained for the $100^{\text {th }}$ and $101^{\text {st }}$ acquisitions with different beamformers. For each of the three adaptive beamformers, two values of adaptive parameter are presented. The green box in (j) is the region of spatial averaging for bias and standard deviation of $v_{z}(x, z)$.

2) Quantitative analysis: For each velocity estimation at a given position $(x, z)$, the bias and standard deviation (STD) of $v_{z}(x, z)$ are computed along the time dimension, considering the 253 velocity maps. These two metrics are spatially 
averaged in the region of interest in the represented green box in Figure 3(j), and normalized with respect to the theoretical maximum axial velocity on the edge of the disk. Results are shown for three rotating speeds of the disk in Figure 4(a) 50 $\mathrm{deg} / \mathrm{s}$, (b) $100 \mathrm{deg} / \mathrm{s}$, and (c) $150 \mathrm{deg} / \mathrm{s}$.

The experimental results demonstrate that advanced beamformers (migration or adaptive) can provide close velocity estimates to those of conventional DAS. The results in Figure 4(b) for the rotating speed of $100 \mathrm{deg} / \mathrm{s}$ are commented. Fourier-based methods slightly outperformed DAS (averaged bias for DAS: $5 \%, \mathrm{Lu}: 4 \%, f-k: 4 \%)$. Adaptive beamformers can offer comparable velocity maps as DAS with similar statistics regarding bias (DAS: 5\%, $\mathrm{MV}_{L=0.3 N}: 5 \%, \mathrm{pDAS}_{p=1.2}$ : $5 \%, \mathrm{PCF}_{\gamma=1}: 6 \%$ ) and STD (DAS: 5\%, $\mathrm{MV}_{L=0.3 N}: 6 \%$, $\mathrm{pDAS}_{p=1.2}: 6 \%, \mathrm{PCF}_{\gamma=1}: 6 \%$ ). However, their performances decreases when increasing the adaptive effect. For example, using pDAS, when the value of $p$ increases, the quality of the velocity estimates is decreasing (bias for DAS: 5\%, $\operatorname{pDAS}_{p=1.2}: 5 \%, \mathrm{pDAS}_{p=2}: 9 \%$, and STD for DAS: $5 \%$, p$\left.\operatorname{DAS}_{p=1.2}: 6 \%, \operatorname{pDAS}_{p=2}: 11 \%\right)$. These trends are preserved, as the rotating speed decreases to (a) $50 \mathrm{deg} / \mathrm{s}$ or increases to (c) $150 \mathrm{deg} / \mathrm{s}$, for all beamformers.
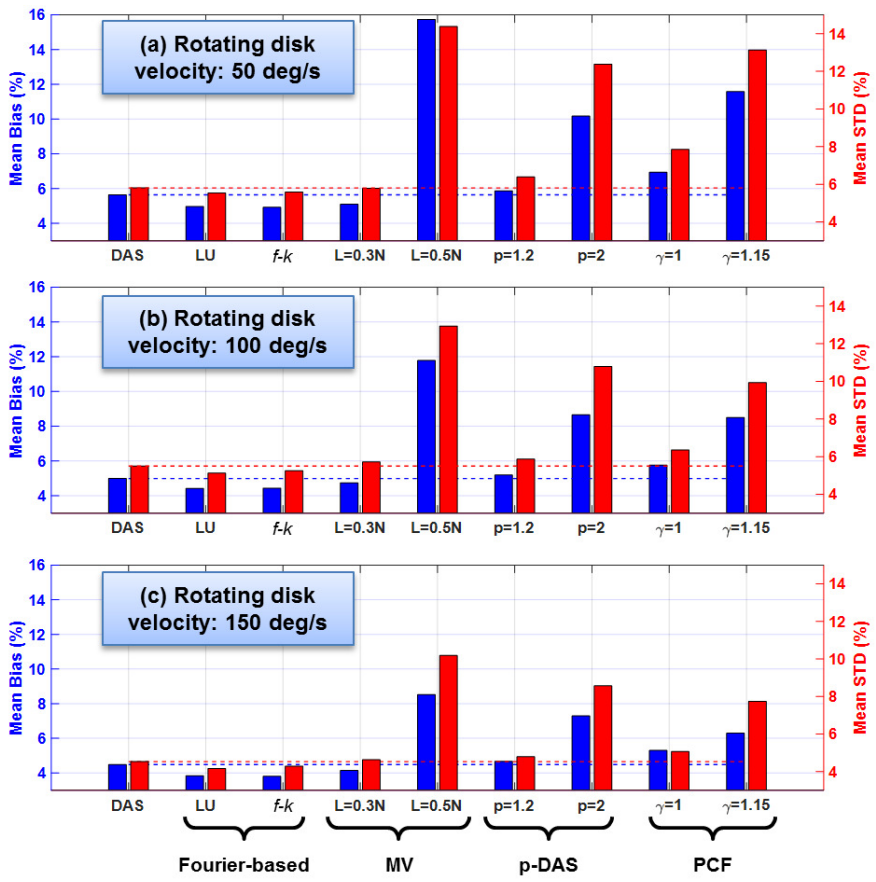

Fig. 4. Normalized biases and standard deviations of axial velocity estimates for different beamforming technics. For each velocity estimation at a given position $(x, z)$, the bias and standard deviation are computed along the time dimension, considering the 253 velocity maps. The metrics are averaged in the region of interest in the green box represented in Figure 3(j). Three rotating speeds were investigated for the disk: (a) $50 \mathrm{deg} / \mathrm{s}$, (b) $100 \mathrm{deg} / \mathrm{s}$, and (c) 150 $\mathrm{deg} / \mathrm{s}$. Metrics are normalized with respect to the theoretical maximum axial velocity of the disk with (a) $13 \mathrm{~mm} / \mathrm{s}$, (b) $26 \mathrm{~mm} / \mathrm{s}$, and (c) $38 \mathrm{~mm} / \mathrm{s}$.

\section{Discussion AND CONCLUSION}

From our knowledge, here is the first experimental and quantitative study on the influence of beamforming methods on velocity estimation. These results demonstrate that advanced beamformers (migration or adaptive) can provide close velocity estimates to those of conventional DAS. A trade-off is at stake between a strong adaptive effect for resolution and contrast improvements of B-mode imaging and degrading the statistics of velocity estimation. These preliminary results pave the way for applications coupling adaptive beamforming with velocity estimations.

\section{ACKNOWLEDGMENT}

This work was supported by the LABEX CELYA (ANR10-LABX-0060) and was performed within the framework of the LABEX PRIMES (ANR-11-LABX-0063) of Université de Lyon, within the program "Investissements d'Avenir" (ANR11-IDEX-0007) operated by the French National Research Agency (ANR).

\section{REFERENCES}

[1] G. Montaldo, M. Tanter, J. Bercoff, N. Benech, and M. Fink, "Coherent plane-wave compounding for very high frame rate ultrasonography and transient elastography," IEEE transactions on ultrasonics, ferroelectrics, and frequency control, vol. 56, no. 3, pp. 489-506, 2009.

[2] M. Tanter and M. Fink, "Ultrafast imaging in biomedical ultrasound," IEEE transactions on ultrasonics, ferroelectrics, and frequency control, vol. 61, no. 1, pp. 102-119, 2014.

[3] J. Cheng and J.-Y. Lu, "Extended high-frame rate imaging method with limited-diffraction beams," IEEE transactions on ultrasonics, ferroelectrics, and frequency control, vol. 53, no. 5, pp. 880-899, 2006.

[4] D. Garcia, L. Le Tarnec, S. Muth, E. Montagnon, J. Porée, and G. Cloutier, "Stolt's fk migration for plane wave ultrasound imaging," IEEE transactions on ultrasonics, ferroelectrics, and frequency control, vol. 60, no. 9, pp. 1853-1867, 2013.

[5] H. Liebgott, A. Rodriguez-Molares, F. Cervenansky, J. A. Jensen, and O. Bernard, "Plane-wave imaging challenge in medical ultrasound," in Ultrasonics Symposium (IUS), 2016 IEEE International. IEEE, 2016, pp. $1-4$.

[6] J. Synnevag, A. Austeng, and S. Holm, "Adaptive beamforming applied to medical ultrasound imaging," IEEE Transactions on Ultrasonics Ferroelectrics and Frequency Control, vol. 54, no. 8, p. 1606, 2007.

[7] M. Polichetti, F. Varray, J.-C. Béra, C. Cachard, and B. Nicolas, "A nonlinear beamformer based on p-th root compression - application to plane wave ultrasound imaging," Applied Sciences, vol. 8, no. 4, p. 599, 2018.

[8] J. Camacho, M. Parrilla, and C. Fritsch, "Phase coherence imaging," IEEE transactions on ultrasonics, ferroelectrics, and frequency control, vol. 56, no. 5, 2009.

[9] T. Loupas, J. Powers, and R. W. Gill, "An axial velocity estimator for ultrasound blood flow imaging, based on a full evaluation of the doppler equation by means of a two-dimensional autocorrelation approach," IEEE transactions on ultrasonics, ferroelectrics, and frequency control, vol. 42, no. 4, pp. 672-688, 1995.

[10] C. Kasai, K. Namekawa, A. Koyano, and R. Omoto, "Real-time twodimensional blood flow imaging using an autocorrelation technique," IEEE Transactions on sonics and ultrasonics, vol. 32, no. 3, pp. 458464, 1985. 